\title{
Menaces et opportunités de l'urbanisation des ports fluviaux. Le cas des métropoles rhénanes
}

Threats and opportunities of urbanizing river ports. The case of the major cities along the Rhine.

Bedrohungen und Opportunitäten bei der Urbanisierung von Binnenhäfen am Beispiel der Metropolen am Rhein.

\section{Antoine Beyer}

\section{OpenEdition}

\section{Journals}

Édition électronique

URL : http://journals.openedition.org/rge/7207

ISSN : 2108-6478

Éditeur

Association des géographes de l'Est

Référence électronique

Antoine Beyer, « Menaces et opportunités de l'urbanisation des ports fluviaux. Le cas des métropoles rhénanes », Revue Géographique de l'Est [En ligne], vol. 57 / 3-4 | 2017, mis en ligne le 12 octobre 2018, consulté le 08 septembre 2020. URL : http://journals.openedition.org/rge/7207

Ce document a été généré automatiquement le 8 septembre 2020

Tous droits réservés 


\section{Menaces et opportunités de l'urbanisation des ports fluviaux. Le cas des métropoles rhénanes}

Threats and opportunities of urbanizing river ports. The case of the major cities along the Rhine.

Bedrohungen und Opportunitäten bei der Urbanisierung von Binnenhäfen am Beispiel der Metropolen am Rhein.

\section{Antoine Beyer}

\section{Introduction}

Les métropoles rhénanes bénéficient d'un remarquable outil logistique. Elles disposent d'importantes emprises portuaires en zones centrales et d'opérateurs fluviaux portés par une activité industrielle de plus en plus tournée vers le grand export. Pourtant cet héritage semble aujourd'hui menacé par les grands projets urbains. Nombreuses sont en effet les municipalités qui sacrifient leurs installations portuaires à la construction de quartiers de standing. Au cours des vingt dernières années, les réalisations emblématiques aux forts accents contemporains se sont multipliées, transformant radicalement les paysages urbains de Duisbourg, Düsseldorf, Cologne ou Mannheim.

Les quartiers neufs conçus par les plus grands architectes du moment satisfont aux injonctions du marketing urbain et modernisent l'image des anciennes cités (Marshall, 2001) (Michon, 2008). Inexorablement, ces évolutions rognent des espaces portuaires qui peinent à se redéployer en périphérie. Les nouvelles réalisations répondent aux besoins effectifs et symboliques qu'exigent les nouvelles fonctions métropolitaines (Wiegmansa et Louwa, 2011), elles relèguent en même temps les fonctions productives et logistiques en marge des agglomérations (Raimbault, 2014) (Dablanc, Frémont, 2015). Or, la voie d'eau, lorsqu'elle est disponible, et c'est le cas pour les villes rhénanes, assure une offre efficace pour l'approvisionnement massifié de l'arrière-pays des 
grands ports maritimes et pour la desserte des cœurs des métropoles (Beyer, Debrie, 2013). Les acteurs portuaires locaux n'ont eu de cesse d'alerter les politiques et l'opinion du danger d'étouffement qui les menace (BÖB, 2006 et 2007).

Le propos de l'article est dès lors de voir comment s'articulent les besoins logistiques et les grands projets urbains dans l'espace rhénan. L'approche soulève les questions suivantes auxquelles nous tâcherons d'apporter un éclairage jusqu'ici peu abordé par les géographes : dans quelle mesure les villes fluviales dupliquent-elles le modèle des waterfronts maritimes (Partie 1) ? Quelle est l'ampleur des mutations portuaires sur les rives du Rhin? Les réaménagements portuaires entrepris sont-ils effectivement de nature à perturber, voire à condamner les activités logistiques (Partie 2) ? Comment les pouvoirs urbains établissent-ils les nécessaires compromis, alors qu'ils sont à la fois porteurs de la stratégie des grands projets urbains et aux commandes des établissements portuaires (Partie 3) ?

Par métropoles rhénanes, nous entendons ici les ensembles urbains de plus de 100000 habitants pour la commune-centre (assurant de fait un rayonnement sur des aires régionales qui avoisinent les 500000 à un million d'habitants) situés au bord du Rhin entre Nimègue à Bâle. Aux 16 villes situées directement sur le fleuve, nous en avons adjoint deux autres sur le Main: Offenbach et Francfort qui sont assimilables aux premières par leur proximité géographique et leur orientation fonctionnelle. Le domaine d'étude s'étend donc des villes néerlandaises de Nimègue et de Arnhem, aux villes d'amont: Strasbourg et Bâle. Ces dernières ayant déjà fait l'objet de recherches (Beyer, 2012 et 2014), elles seront ici moins détaillées au profit d'étude de cas allemands. Pour chacune des villes, la démarche a constitué à prendre connaissance des projets urbains, réalisés ou en cours de développement, à partir des publications scientifiques, et des documents de planification officiels. Partant de la confrontation systématique de ces sources, notre ambition est de proposer un modèle rhénan de reconquête urbaine des espaces portuaires questionnée à l'aune des waterfronts maritimes. Une connaissance précise des situations individuelles par des visites de terrain nous a permis par ailleurs de compléter et d'illustrer notre propos.

\section{Du maritime au fluvial, le modèle urbain des fronts d'eau}

\section{Jusqu'à quel point peut-on appliquer la notion de waterfronts aux villes fluviales?}

Les ports fluviaux s'inscrivent dans les tendances historiques d'éloignement spatial et fonctionnel de la ville et de ses activités, telles qu'en rendent compte les schémas de Bird (1971), de Hoyle (1989) et de Merckx.et al. (2003). Si le phénomène de déconnexion (Hall \& Clark, 2010) a été très précoce et intense dans le cas des ports maritimes avec la notion de water front redevelopment (Breen et Rigby, 1996), ils interviennent plus tardivement pour les ports fluviaux où le foncier est plus rare et le lien plus étroit avec les acteurs politiques et économiques urbains. Dans les deux configurations, la multiplication des friches concerne les parties les plus anciennes et les plus centrales des installations portuaires. Cette évolution est la conséquence d'un ajustement à l'environnement économique et à l'essor du transport conteneurisé. Les zones laissées en déshérence vont alors intéresser les urbanistes pour des usages largement étrangers 
au fret et à sa transformation : promotion de la question environnementale en ville, requalification urbaine des berges, valorisation patrimoniale d'éléments industriels menacés, enfin et surtout, capacité de redéployer des fonctions centrales dans un espace ouvert aux réalisations architecturales contemporaines (Charlier, 2007).

Issus du vocabulaire anglo-saxon, les termes de "waterfronts» et de "régénération urbaine " sont communément appliqués à ces grandes opérations. Les réalisations portuaires les plus précoces ont concerné les métropoles nord-américaines dès les années 1970 avec les Inner Harbour de Boston et de Baltimore, le Battery Park à New York, le nouveau Downtown à Vancouver (Chaline, 1994) (Wang, 2002) (Schubert, 2011). Le mouvement a touché l'Europe au cours de la décennie suivante avec les Docks de Londres, les Docks Albert à Liverpool, l'Art District, de Bilbao et son célébrissime Guggenheim Museum, ou encore le Kop van Zuid à Rotterdam. Au sud du continent, les réaménagements portuaires ont été entrepris à l'occasion de grands événements internationaux : les Jeux Olympiques à Barcelone (1992), les expositions universelles à Gênes (1992) et de Lisbonne (1998). Plus récemment, on peut citer la réalisation de la Hafenstadt à Hambourg ou le projet EuroMéditerranée à Marseille. Les innombrables publications sur le sujet soulignent l'impact qu'ont pu avoir chacune de ces réalisations. Leur expansion géographique (Brownhill, 2013) et les variations thématiques ont conduit à en renouveler l'analyse sous l'angle du tourisme et de la ville créative (Sepe, 2013) ainsi que de la question environnementale (Dyson \& Yocom, 2015). Lieux d'innovation urbanistique, les waterfronts maritimes ont indéniablement marqué l'imaginaire collectif, autant du grand public que des aménageurs.

Le principe de ces divers projets est la valorisation des éléments de centralité. Les fonctions de direction y sont privilégiées, car le réaménagement des anciennes zones portuaires doit assurer l'émergence de quartier à vocation métropolitaine. Ce dernier doit accélérer le passage d'une économie urbaine encore marquée par des fonctions industrielles à une économie de services. La forte mobilisation de capitaux privés dans le montage des opérations se traduit dans le choix des aménagements : forte densité du bâti, réalisations de prestige pour attirer les clients les plus solvables, programmes marqués par les thèmes de la sécurité et par l'encadrement étroit des espaces publics et de leurs usages. On retrouve là les phénomènes associés au processus de gentrification (Smith, 2002), d'autant que la question de la mixité sociale est volontairement reléguée au second plan. Le prestige symbolique des sites est de fait attesté par la préservation de quelques bâtiments historiques et la promotion d'une atmosphère portuaire largement réinterprétée (Malone, 1996) (Brownhill, 2013). La conception néolibérale de ces nouveaux espaces urbains a été très fortement soulignée par les approches critiques qui ont vu dans les régénérations portuaires le laboratoire d'un mode de gestion qui s'est ensuite diffusé (Gintrac, Giroud, 2014). En d'autres termes, ces nouveaux quartiers fonctionnent comme des vitrines de la ville globale financiarisée mise en avant par Saskia Sassen (1996).

Le caractère ostentatoire d'une mutation assumée trouve un écho dans l'architecture postmoderne qui s'impose à la même époque sur la scène internationale (Jencks,1984). Elle revendique une rupture avec l'approche fonctionnaliste et productiviste prônée par le mouvement moderne et soutient le retour à des références stylistiques plus classiques, même si elles sont en partie détournées et rendues spectaculaires. Dans un contexte concurrentiel accru, cet urbanisme investit pleinement la dimension de marketing urbain pour attirer des fonctions de commandement. La réalisation de 
bâtiments de prestige à l'architecture soignée (musée, opéra, philharmonie) est alors un élément-clé de promotion et de valorisation des nouveaux quartiers portuaires dont ils constituent les signaux forts. Bref, les nouveaux fronts d'eau relèvent d'un discours et d'une pratique urbaine libérale assumée. Ils sont pour les édiles et les classes dirigeantes, tout à la fois le signe d'une ville capable de se réinventer et le lieu d'une résidence "exclusive » dotée de toutes les aménités. Un morceau de ville accessible certes, mais sous contrôle, affichant les valeurs de l'entreprenariat et résolument tournée vers les activités d'encadrement et accueillant des activités de loisirs.

Dans quelle mesure alors l'interprétation socio-spatiale proposée des fronts d'eau maritimes peut-elle s'appliquer à l'espace urbain des villes du Rhin ? La société rhénane n'est-elle pas, à l'instar de son capitalisme, jugée comme socialement plus intégratrice via le fameux capitalisme rhénan et l'ordo-libéralisme ? Cette lecture conduirait à atténuer les effets les plus ségrégatifs des intérêts financiers en jeu tels qu'ils s'expriment dans un contexte anglo-saxon. Par ailleurs, comment un espace où l'activité industrielle reste très présente peut-il renoncer au formidable potentiel de transport que représente le Rhin pour s'orienter vers des activités à forte dominante tertiaire? Il s'agit dès lors de présenter la manière dont les références paysagères et fonctionnelles mobilisées sont présentes dans les réalisations rhénanes, de manière implicite ou explicite.

\section{Les dynamiques métropolitaines au cœur des mutations portuaires sur le Rhin}

La proximité de l'eau est indéniablement devenue un élément moteur de l'urbanisation contemporaine (Holländer et al., 2011) et cela s'applique aussi aux villes rhénanes. Comme pour le littoral, elle est associée à des valeurs positives car donnant accès aux aménités urbaines: les berges dégagées et transformées offrent des promenades, ouvrent des perspectives paysagères inhabituelles à proximité des hyper-centres. Les programmes d'équipement promettent la réduction des distances au lieu de travail et à la centralité urbaine. L'association des fonctions apparaît clairement dans le slogan de promotion repris par ces grandes opérations immobilières : habiter et travailler au bord de l'eau, "Wohnen und arbeiten am Wasser" que l'on retrouve dans nombre de slogans de promotion immobilière.

Les nouveaux quartiers sont dotés d'institutions culturelles phares et offrent d'autres types de loisirs sportifs ou gastronomiques. Nombre de ces bâtiments remarquables mais difficilement convertibles en logements du fait de leur structure, sont alors dédiés à des usages culturels ou muséaux, pouvant le cas échéant rappeler les orientations passées du lieu : le Musée du chocolat et le Musée du sport et de l'olympisme à Cologne, le musée historique de la ville à Duisburg, le Musée des beaux-arts à Mayence, la médiathèque à Strasbourg qui est hébergée dans les anciens entrepôts Seegmüller. Diverses activités culturelles et ludiques trouvent des expressions plus modestes à bord de bateaux amarrés. Cette offre vient compléter et diversifier des dispositifs souvent déjà riches et assurés par les institutions du centre-ville, grâce à une programmation plus libre voire alternative.

Comme dans les ports maritimes, pour lancer les réalisations, les urbanistes convoquent de prestigieuses signatures architecturales (Ponzini et al., 2011) sur le modèle de Bilbao. Les villes cherchent en effet à s'assurer une réalisation iconique, un 
emblème auquel rattacher le discours de promotion. Le geste architectural fort vise au rajeunissement de l'image de la ville, liant l'audace et la créativité. Architectes et urbanistes passent ainsi des ports maritimes aux ports fluviaux. La verticalité d'immeubles belvédères et les réminiscences historiques des grues médiévales caractérisent le projet du Waalhaven de Nimègue. On retrouve les réalisations emblématiques de Franck Gehry dans le Medienhafen de Düsseldorf. Dans le port urbain de Duisbourg (Innenhafen), on retrouve plusieurs réalisations remarquables : celle de Nicholas Grimshaw avec le Hitachi Power office, le musée des beaux-arts de Herzog \& de Meuron ainsi qu'un bâtiment arqué, d'un blanc immaculé conçu par l'architecte britannique, Norman Foster. L'édition 2009 du MIPIM a couronné les singuliers immeubles en porte-à-faux d'Alfons Linster et Teherani à Cologne, les désormais fameux Kranhäuser, groupe de trois immeubles de $60 \mathrm{~m}$ de haut en forme de grues. Leurs silhouettes se sont inscrites dans le paysage urbain de Cologne à côté des célèbres flèches de la cathédrale (Fig. 1).

Figure 1. Les immeubles-grues (Kranhäuser), emblématiques de la silhouette urbaine de Cologne (Source Wikipédia commons).

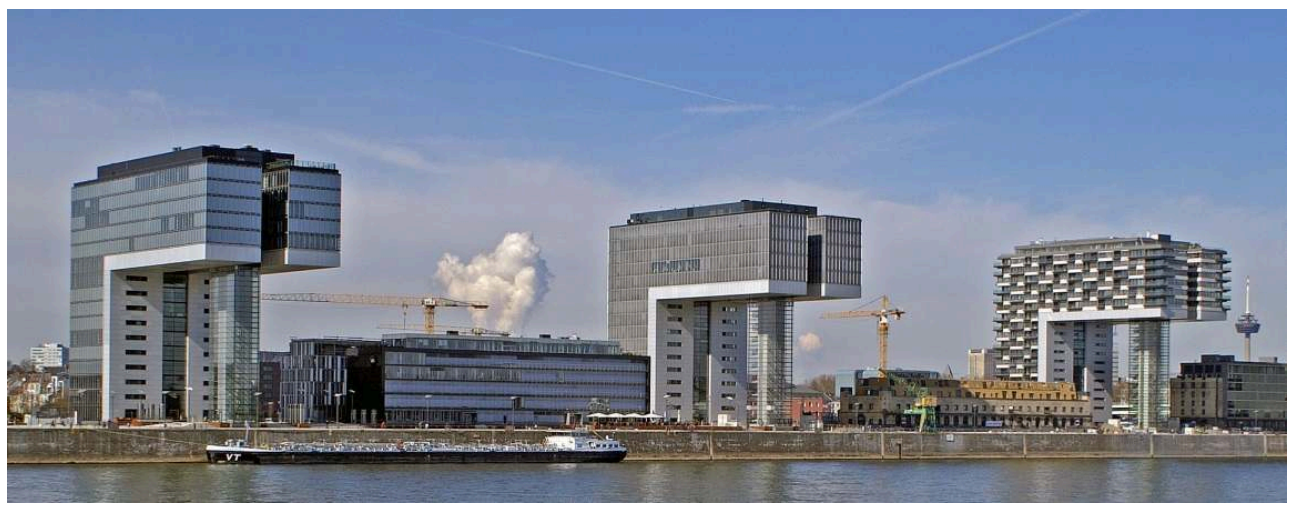

L'effet Guggenheim reproduit sur les rives du Rhin joue à plein. Il traduit une réelle émulation entre villes et encourage la réalisation de projets toujours plus ambitieux et singuliers. Les nouveaux quartiers peuvent d'emblée être dédiés à des effets de clustering, autour des médias à Düsseldorf, de l'art et des médias à Cologne, autour de la finance à Francfort ou encore des biotechnologies à Bâle. L'ensemble le plus spectaculaire est indiscutablement la concentration de bâtiments à Bâle en lieu et place de l'ancien port Saint-Jean et dans son immédiate proximité. Il fonctionne comme un véritable musée de l'architecture contemporaine à ciel ouvert au sein du Campus Novartis. Les réalisations sont aussi parfois moins éclatantes. Ainsi, porté par l'urbaniste catalan Manuel de Solá-Morales, initiateur de la reconquête des friches portuaires de Saint-Nazaire (1998-2002), l'ambitieux quartier culturel du Rijnboog qui visait à recréer une centralité urbaine à Arnhem, a été revu à la baisse. De leur côté, les villes de Strasbourg et de Mannheim sont restées plus modestes dans leurs réalisations.

La requalification urbaine des espaces portuaires s'accompagne d'une profonde transformation sociale des quartiers concernés. Les nouveaux quartiers sont avant tout conçus pour les classes favorisées avec une surreprésentation d'appartements de standing, largement ouverts par des baies vitrées, de vastes terrasses donnant sur d'anciens bassins improvisés en marinas. A Cologne, le quartier du Rheinauhafen enregistre des prix qui sont trois fois plus élevés que la moyenne de la ville. Il en résulte des ambiances urbaines un peu mornes et socialement homogènes (Höhmann, 
2010). Cette ségrégation s'inscrit par ailleurs en Allemagne dans une dynamique générale de baisse de la part du logement social dont le nombre est tombé de 3 millions dans les années 1990 à 1,5 million aujourd'hui (Hendricks, 2017). A contrario, pour la réhabilitation du Deutzer Hafen lancée en 2016, la municipalité de Cologne annonce officiellement un tiers de logements sociaux, ce qui s'avère être une exception à la fois par son affichage et par son importance (Stadt Köln, 2016). On retrouve aussi cette préoccupation à Strasbourg avec le projet des Deux rives qui assure la réhabilitation du quartier très social du Port du Rhin et la mixité dans les nouvelles constructions (Ville de Strasbourg, 2010). En contrepartie, la valorisation des espaces-(virgule à supprimer) conduit à une spéculation sur les quartiers voisins, jusque-là dévalorisés par les nuisances liées aux activités portuaires ou aux logements ouvriers. Mais là encore, l'encadrement politique et réglementaire entre en jeu qui tempère le caractère ségrégatif des nouvelles réalisations. Aussi, au-delà des simples artefacts visuels et des codes architecturaux qui semblent indiquer des effets systématiques de gentrification, des analyses plus précises mériteraient d'être menées systématiquement.

L'aménagement relève souvent d'un partage des rôles entre le public et le privé. A Mayence, la municipalité (à travers sa régie, les Mainzer Stadtwerke) s'investit dans la réhabilitation des bâtiments historiques pour accueillir un musée des beaux-arts, un centre dédié au vin dans les anciens entrepôts (Weinerlebniszentrum) alors que les promoteurs privés sont, pour leur part, engagés dans l'offre de logements.

Une spécialisation fonctionnelle est envisageable dès lors que les surfaces disponibles sont importantes. Ainsi, le projet urbain de Ludwigshafen vise à revitaliser le centreville en l'ouvrant aux rives du Rhin dont il était jusque-là coupé par les emprises industrialo-portuaires. Il intègre pour cela deux espaces (Fig.2) :

- l'ancien Zollhofhafen (premier tiers du XIXe siècle) accueille de nouvelles fonctions de centralité qui lui faisaient défaut : un espace marchand de $30000 \mathrm{~m} 2$ est inauguré en 2010, la Rhein-Galerie, accroissant de 50\% l'offre de surface commerciale du centre-ville. L'ancienne halle du chantier fluvial est transformée en pépinière pour l'économie numérique ; un pôle hôtelier et de restauration est créé autour du bassin Luitpold transformé en marina. L'ensemble est complété par des attractions culturelles (edutainment).

- Au sud, sur 31 hectares, la mutation touche des espaces dévolus jusque-là aux activités industrielles (Rheinufer-Süd). Elle permet un développement aéré, orienté vers l'offre résidentielle (700 logements), des petits collectifs mais aussi des villas urbaines associées à des services de proximité. Les rives du Rhin elles-mêmes sont requalifiées en promenades paysagées. Les activités portuaires ont quitté les bassins centraux en 2004 pour être relocalisées au nord Kaiserwörthhafen. Cette opération d'ampleur a pour objectif de repositionner Ludwigshafen à l'image plus ouvrière face à sa voisine et rivale Mannheim, tant dans l'offre commerciale que pour le nouveau standing de son offre immobilière. 
Figure 2. Ludwigshafen. Carte de localisation des sites portuaires urbanisés

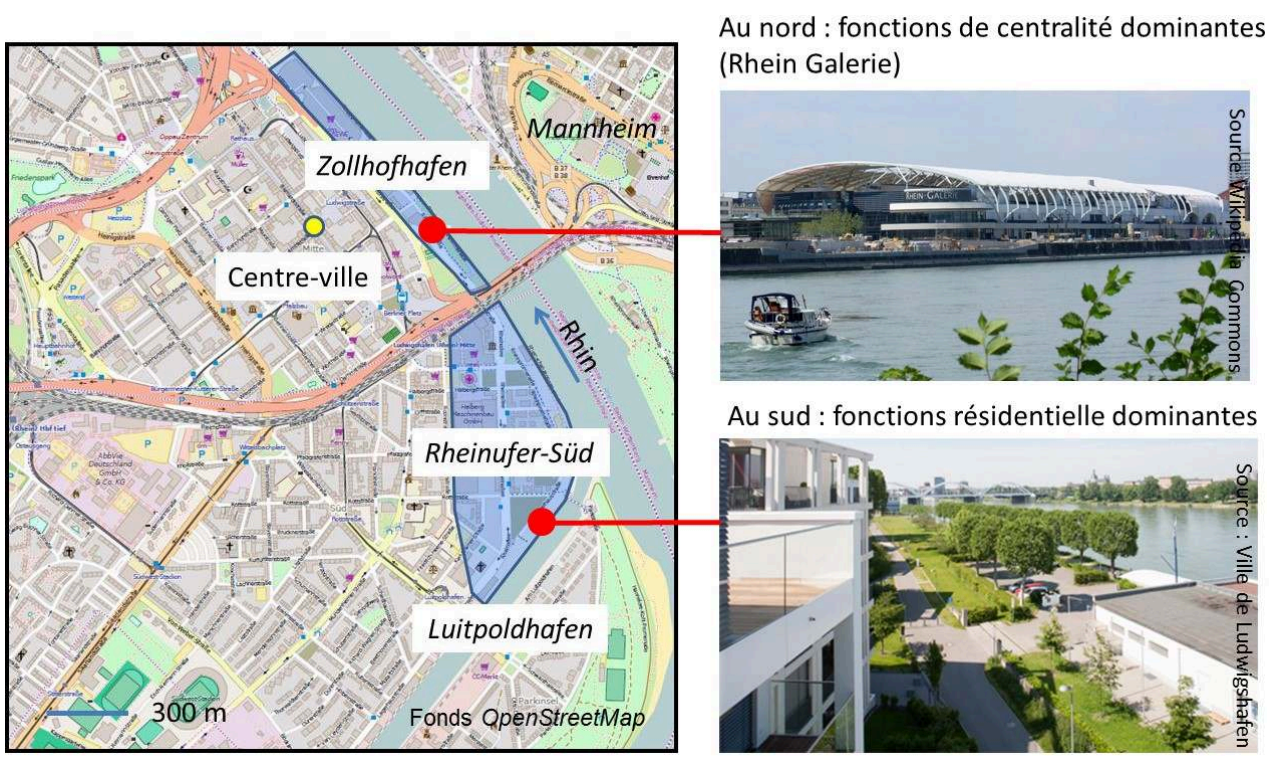

Le cadre urbain des villes rhénanes est ainsi progressivement métamorphosé. Il accompagne un changement de valeur plus profond, où le cadre urbain s'éloigne du monde du labeur industriel et la manutention portuaire, du bruit et de la poussière. Cette réalité passée est alors transfigurée par les emblèmes que sont les cheminées éteintes, les vieilles grues à usage décoratif ou les entrepôts transformés en galeries marchandes. Les anciennes zones portuaires s'ouvrent sur des lieux apaisés où le monde traditionnel du travail physique est relégué à l'arrière-plan, comme pour mieux souligner que la valeur ajoutée passe désormais par d'autres canaux.

\section{Ampleur et formes des mutations portuaires rhénanes}

\section{L'estimation quantitative du phénomène de requalification}

L'évolution des logiques productives et le renforcement de la concurrence internationale inscrivent les acteurs publics et privés dans un processus de métropolisation dont la mutation des espaces portuaires est le théâtre. Toutefois, les champions industriels allemands, des PMI aux grands groupes restent les leaders dans bien des domaines et, pour maintenir leur rang, ils sont poussés à investir tout à la fois dans la R\&D et la logistique pour accéder à de nouveaux marchés. La reconquête des espaces portuaires centraux offre ainsi un potentiel facilement mobilisable tout au répondant au mieux aux attentes du marché. Le nombre et l'ampleur des opérations récentes de ce type sont notables. La quasi-totalité des pôles urbains de plus de 100000 habitants pour la commune-centre ont mis en place un programme de reconversion de leurs espaces portuaires au cours de la décennie passée (Tab. 1). 
Tableau 1. Ampleur des espaces de reconversion urbaine des emprises portuaires dans les métropoles rhénanes (Conception de l'auteur, données des diverses administrations portuaires et municipales).

\begin{tabular}{|l|l|l|l|l|l|l|l|l|}
\hline & $\begin{array}{l}\text { Taille de la } \\
\text { commune } \\
\text { (en 1000 } \\
\text { hab.) }\end{array}$ & $\begin{array}{l}\text { Trafic } \\
\text { total } \\
\text { (en 1000t) } \\
2015\end{array}$ & $\begin{array}{l}\text { Trafic de } \\
\text { conteneurs } \\
\text { tous modes } \\
\text { Ports }\end{array}$ & $\begin{array}{l}\text { Superficie } \\
\text { actuelle du } 1000 \text { TEU) } \\
\text { port (ha) }\end{array}$ & Projet urbain & $\begin{array}{l}\text { Année de } \\
\text { lancement }\end{array}$ & $\begin{array}{l}\text { Taille de } \\
\text { l'operation } \\
\text { (ha) }\end{array}$ & $\begin{array}{l}\text { Disponibilité } \\
\text { fonciere pour } \\
\text { l'extension du } \\
\text { port (2014) ha }\end{array}$ \\
\hline Amhem & 156 & - & - & - & Rijnboog & 2001 & 80 & - \\
\hline Bâle & 166 & 6334 & 124 & 125 & New Basel & 2005 & 18,7 & 0 \\
\hline Bonn & 310 & 620 & 209 & 63 & Non & - & - & 12 \\
\hline Duisbourg & 488 & 52423 & 3583 & 933 & Innenhafen & 1995 & 50 & 15 \\
\hline Dusseldorf & 601 & 195 & 171 & 150 & Medienhafen & 1989 & 26 & 50 \\
\hline Francfort \\
Main)
\end{tabular}

Dans son travail de recherche, Uwe Stöckner estimait en 2005 déjà que $60 \%$ des restructurations portuaires allemandes sur le Rhin et le Neckar étaient réalisées pour renforcer la centralité urbaine, $30 \%$ pour réhabiliter les rives du fleuve et $14 \%$ seulement pour consolider les fonctions portuaires. Si l'on se limite maintenant aux principales villes, l'analyse détaillée des programmes d'aménagement que nous avons menée permet d'identifier plusieurs types d'attitudes quant à la justification des réaménagements portuaires :

- répondre aux besoins de logements (Francfort, Düsseldorf, Bâle)

- susciter l'installation de fonctions directionnelles - ou d'activités à haute valeur ajoutée (Bâle : biotechnologies - Francfort : activités financières dont BCE).

- relance de l'économie et besoin de redorer une image par des aménités (Duisburg, Ludwigshafen)

- volonté de limiter la périurbanisation (Strasbourg- Bâle), d'étendre (Nimègue) ou de recréer un centre-ville (Arnhem).

Si certaines villes envisagent d'amputer les surfaces portuaires sans compensation, surtout celles où la pression est la plus forte (Francfort, Düsseldorf), nombreuses sont celles qui accompagnent le transfert d'activités pour moderniser les installations. A Strasbourg, les anciens entrepôts ont été rasés pour laisser la place à un terminal de conteneurs au moment où les espaces plus urbains sont dévolus aux opérations immobilières. Les extensions modernes du port ont été réalisées à Lauterbourg à $30 \mathrm{~km}$ plus au nord. A Mayence, le Zollhafen ${ }^{1}$ a fait l'objet d'une opération d'urbanisation sur 22 hectares. Pour ce faire et dès 2003, les activités logistiques ont été renforcées en aval dans le cadre d'un financement fédéral pour la promotion de chantiers intermodaux. 
Les chantiers sont lancés en 2011, après le déménagement définitif du port intervenu l'année précédente.

Les réalisations urbaines se lisent aussi dans le dynamisme qu'ils elles insufflent au marché local de l'emploi. Düsseldorf aurait ainsi bénéficié de la localisation de 8400 postes de travail au Medienhafen (Düsseldorf); à Cologne le Rheinhauhafen en aurait suscité 2100 et l'on annonce 6000 emplois à Deutz. La ville de Duisbourg ne compte pas moins de 5000 emplois dans les zones reconverties de l'Innenhafen (NRW, 2010). A Bâle, les plans directeurs du nouveau quartier des Trois-Pays (Drei-Länder Quartier) envisagent à terme d'accueillir 20000 emplois.

\section{Les configurations rhénanes de la régénération portuaire}

Le phénomène de régénération portuaire touche de manière différenciée les espaces portuaires, selon leur degré de centralité et la puissance industrielle des métropoles concernées. Un modèle graphique (Fig.3) permet de rendre compte de différentes évolutions possibles observées dans les principaux ports rhénans. La cas générique ou Anyport pour reprendre la terminologie proposée par Bird (1971) rappelle le caractère commun du fleuve dans la dynamique urbaine et portuaire. La présence du Rhin introduit en effet un développement urbain dissymétrique. On distingue alors le noyau historique (Altstadt) établi sur une des berges du fleuve au profit parfois d'une confluence (A). Les développements urbains ultérieurs sont définis comme les banlieues denses ou Neustadt (B). Enfin, l'extension contemporaine de l'unité urbaine, l'espace métropolitain ou Metropolregion (C) s'étale sur les deux rives du fleuve à la faveur de la multiplication des ponts et de l'usage généralisé de l'automobile.

Dans les principales villes rhénanes, les emprises portuaires sont historiquement éclatées. La situation traduit les différentes étapes de leur mise en place. L'implantation initiale remonte souvent au Moyen-Âge (Cologne, Francfort), parfois à l'époque moderne (Mannheim). Elle privilégie une position centrale, car susceptible de répondre au mieux à une fonction commerciale (Stadthafen). Dans la seconde moitié du XIXème siècle, l'extraordinaire essor de la navigation industrielle conduit à développer des équipements et à spécialiser les espaces. Les installations centrales sont alors aménagées pour accueillir les envois manufacturés et les produits de plus forte valeur avec les capacités d'entreposage sous douane, alors qu'en périphéries apparaissent les ports industriels (Industriehafen) qui seront progressivement rattrapés par l'urbanisation. Au tournant du XXe siècle, de nouveaux investissements sont réalisés : bassins et réserves foncières sont constitués aux marges des extensions urbaines. Les dernières extensions, lorsqu'elles sont lancées, sont réalisées au lendemain de la Seconde Guerre mondiale et jusque dans les années 1960. Elles accompagnent le développement en périphérie plus lointaine de certains trafics spécialisés comme les hydrocarbures à Cologne-Godorf ou à Karlsruhe (raffinerie Miro), plus récemment avec des terminaux à conteneurs (Nimègue). Constitués en sociétés de droit commercial avec des capitaux municipaux, les ports rhénans disposent d'une large autonomie de gestion qui leur a permis de développer des chantiers de transport multimodaux au cours de la décennie 1990 - 2000, avec l'appui des politiques fédérales. Les plus importants d'entre eux disposent même de leurs propres filiales ferroviaires (Beyer, 2012.2). Ces mouvements conduisent à des développements par substitution des activités en déclin (brown field) ou plus rarement d'aménagement au détriment d'espaces naturels ou 
agricoles (green field) - correspondant à la catégorie " expansion ». Enfin, la situation de « statu quo » traduit la stabilité des emprises et correspond à des villes sans port urbain en position de centralité. Les ports qui réduisent leurs emprises portuaires sans compenser ces pertes peuvent être rangées dans la catégorie «amputation ». Souvent le Rhin coïncide avec les circonscriptions administratives : limite de communes, de Länder et plus en amont d'Etats.

Figure 3. Structures-types des installations portuaires des villes rhénanes. (Conception A. Beyer)
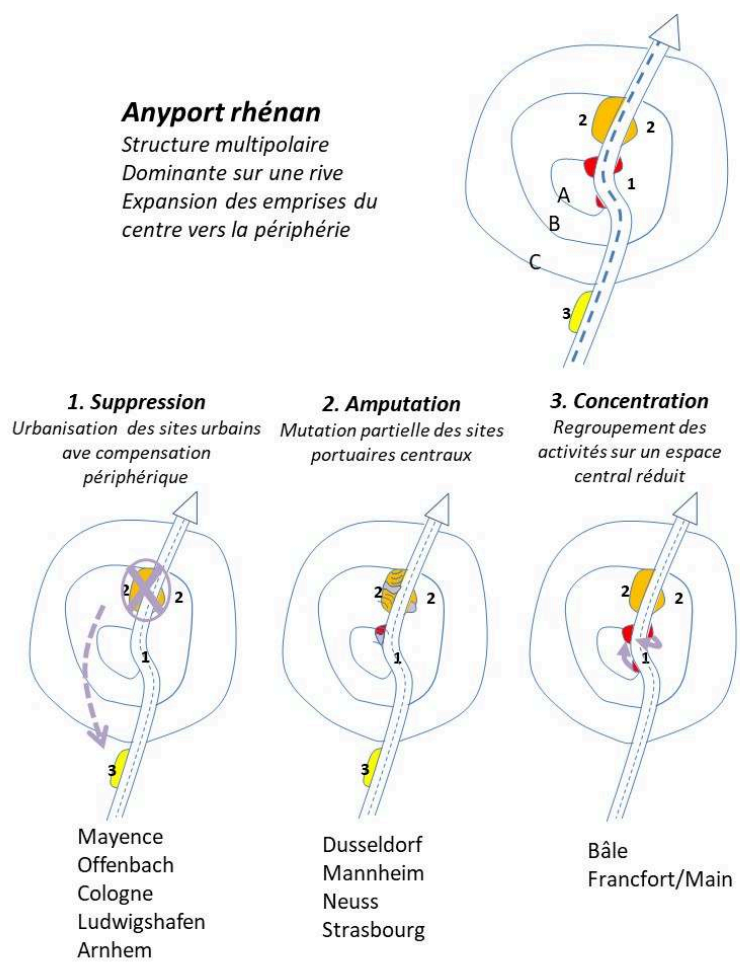

Limites administratives

1. Port urbain (Stadthafen)

2. Port industriel (Industriehafen)

3. Nouveaux terminaux en périphérie

A. Cœur urbain

B. Banlieues denses

C. Espace métropolitain
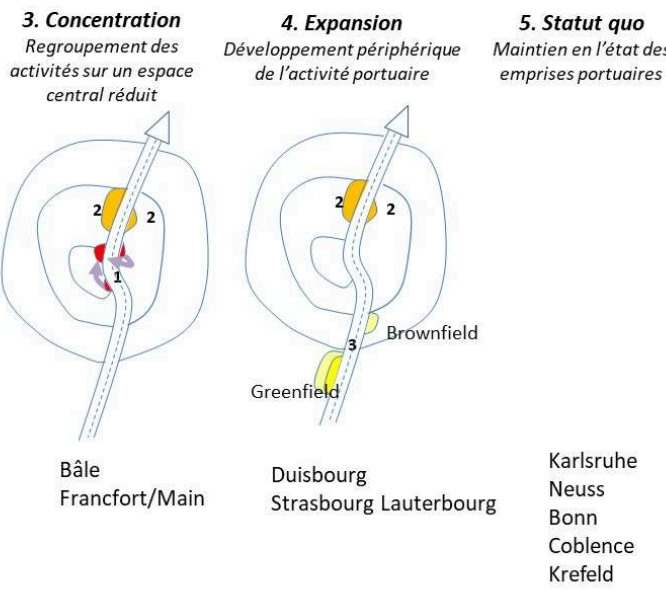

Ainsi, en fonction des opportunités foncières, des incorporations communales et des coopérations commerciales, le développement des ports s'est opéré de manière discontinue. C'est donc dans un système portuaire relativement éclaté qu'ont lieu les restructurations, les pressions urbaines les plus fortes s'exerçant sur les implantations centrales. Ces dernières, si elles sont les plus convoitées, sont aussi les moins nécessaires au traitement des trafics. Les ports rhénans partagent ici des caractéristiques avec d'autres ensembles fluviaux (Frémont, 2012) (Paffoni 2013) (Debrie \& Raimbault, 2016). Ces extensions urbaines successives remettent-elles alors en cause les orientations des installations portuaires et permettent-elles de comprendre comment sont négociées de telles mutations fonctionnelles?

\section{La construction de nouveaux équilibres}

\section{Comment accompagner la mutation fonctionnelle des espaces portuaires?}

Après avoir abordé la question en termes spatiaux, il convient d'envisager les relations plus institutionnelles qui accompagnent la redéfinition de la relation ville-port. Il faut 
d'abord rappeler qu'à notre connaissance, aucune municipalité n'est foncièrement opposée au maintien de l'activité portuaire en milieu urbain. Dans certain cas, au contraire, la décision peut lui être favorable comme c'est le cas avec la municipalité de Neuss qui a rejeté un projet de requalification urbaine d'espace portuaire et la construction de bureaux au profit d'une activité logistique (NRW, 2010). La priorité accordée à l'activité portuaire participe alors d'une orientation stratégique globale et d'une position assumée en faveur de l'activité portuaire. A l'opposé, la Ville de Düsseldorf s'est engagée dans la substitution progressive de son activité portuaire au profit de services à haute valeur ajoutée sans rapport (sinon paysager) au fleuve. Le succès du projet Medienhafen l'incite à envisager des extensions au détriment des espaces portuaires voisins (Fläming, Hesse, 2010). Le déclin des trafics et l'obsolescence des aménagements portuaires permettent d'envisager une transition fonctionnelle douce. Souvent, les espaces urbanisés ont perdu toute activité portuaire de longue date: l'étroitesse des bassins et leur insuffisante profondeur n'autoriseraient une adaptation aux conditions actuelles d'exploitation qu'au prix de très lourds travaux. Il y a ainsi dans la reconversion une mutation naturelle et bienvenue des espaces confrontés à l'obsolescence. Uwe Stöckner estime que cette situation représente $60 \%$ des cas qui pour la moitié d'entre eux ne traitent déjà plus de trafic (Stöckner, 2005, p. 112).

A Bâle, c'est l'ensemble du site portuaire Saint-Jean qui a cédé sa place au campus Novartis. Peu accessible par route ou rail, il imposait la traversée de quartiers résidentiels par des chargements de fret. Son intérêt s'était au demeurant fortement réduit alors que la vocation industrielle du quartier s'effaçait au profit d'une orientation R\&D. La taille des parcelles et leur enclavement, adaptés à des fonctions de transbordement tel qu'il se pratiquait dans le passé, se révèlent insuffisants. Le port de Münster sur le canal Dortmund-Ems a connu un déclin tel que l'activité portuaire était condamnée. C'est ainsi la totalité de l'emprise portuaire qui a fait l'objet d'urbanisation avec le lancement du projet Kreativ Kai. Le port historique de Duisbourg, l'Innenhafen a perdu depuis trente ans toute vocation fluviale: à quelques kilomètres de l'artère rhénane, il exigeait le franchissement d'une étroite écluse. Par ailleurs, les quelques 50 hectares du site ne représentent que 3,5\% du domaine portuaire. De toute façon, la patrimonialisation de divers bâtiments industriels en aurait interdit une modernisation efficace. Pour tirer parti de l'engouement des logements bord à quai, le creusement d'un nouveau bassin adjacent au port (en fait plutôt une pièce d'eau peu profonde) a même permis d'accroitre le potentiel de terrains à lotir avec vue sur l'eau. Les aménageurs ont suivi ce même procédé à Mayence en doublant un canal.

Dans bien des cas, c'est l'autorité portuaire qui entame volontairement le processus de déclassement. L'urbanisation traditionnelle n'est cependant pas la seule option à envisager pour définir un nouvel usage aux espaces portuaires en déshérence. D'autres activités plus liées à l'ouverture fluviale des espaces peuvent être promues. On pense bien sûr aux terminaux de croisières fluviales qui bénéficient des infrastructures existantes et de la proximité potentielle du centre urbain historique. Le port de Strasbourg a ainsi envisagé un terminal de croisière fluviale relié au centre par tramway sur un môle dédié autrefois au transbordement du charbon. A Köln-Deutz, la contre-proposition, finalement non-retenue de la création d'un quartier avec marina, consistait à maintenir les ateliers de maintenance des bateaux de croisière, très nombreux à jeter l'ancre à Cologne. Cette option présentait l'avantage d'être réversible et de conserver des emplois et un savoir-faire directement lié à l'économie du fleuve. 
Toutes les zones portuaires ne sont pas aptes à accueillir de l'urbanisation du fait des risques d'inondation. Le Project Blau Mannheim destine l'essentiel des réaffectations foncières à l'usage récréatif et à la renaturalisation des berges reconquises (promenades, parcs, pistes cyclables). Mais, comme on a déjà pu le voir, l'argument des risques d'inondation ne constitue pas un frein absolu, dès lors que des mesures adéquates sont prises : renforcement des digues, emprise réduite au sol pour faciliter la circulation des eaux, rez-de-chaussée réservés à des fonctions de parking.

Jusqu'à un certain point, la réduction des surfaces portuaires n'est pas synonyme de baisse de trafic, à la condition que des efforts de productivité soient réalisés sur des quais restants. Les variations de tonnages manutentionnés par mètre linéaire sont considérables (de 1 à 7 selon Stöckner 2005, p. 97) et laissent envisager d'importants gains de productivité. Tel a été notamment l'option retenue à Francfort sur le Main où les trafics du Westhafen ont été réorientés vers les sites voisins de Osthafen ou de Gutleuten Hafen. La centrale thermique dont le charbon est acheminé par voie d'eau n'aurait pu être transférée sans un coût économique prohibitif. Il a donc été imaginé un système de transbordement par voie transporteuse depuis les quais, remarquablement intégré dans le nouveau quartier.

La cession du foncier portuaire et sa valorisation urbaine représente une opportunité financière qui peut permettre au port un redéploiement et une modernisation sur d'autres sites, difficiles sinon à réaliser. L'investissement ne concerne alors pas que les aménagements fluviaux mais conduit à renforcer l'efficacité portuaire par l'amélioration des dessertes routières et ferroviaires (développement des voies ferrées portuaires, aménagement du chantier de transport combiné etc.). Certaines autorités portuaires ont d'ailleurs fait le choix d'assurer eux-mêmes la fonction de promoteurs fonciers, voire d'en conserver au moins partiellement la propriété. Cela leur assure une participation aux profits d'un droit de regard sur les aménagements réalisés permettant de garantir la compatibilité du nouvel usage avec les fonctions portuaires existantes. Tel a été le choix de l'autorité portuaire de Cologne où l'opération d'urbanisation du Rheinauhafen a été portée par la société portuaire (HGK - Häfen und Güterverkehr Köln). Par son association avec des promoteurs immobiliers, le Port Autonome de Strasbourg a aussi retenu la solution du bail à long terme qui lui assure un revenu régulier en lui garantissant la propriété du foncier.

A l'usage, il s'avère que le choix de la mixité des fonctions en cas de réhabilitation est celui qui est plus difficile à gérer. Les conflits d'usage ne manquent pas d'apparaître entre les exigences des nouveaux riverains qui ont acquis souvent cher leur bien et l'activité bruyante, parfois polluante qui continue à s'exercer à proximité. Ces situations ont pu conduire les gestionnaires portuaires à développer des zones d'activités de transition, ou zones tampon où les activités exercées émettent moins de nuisances tout en tolérant des activités industrielles traditionnelles. Des investissements spécifiques peuvent être engagés pour permettre certains voisinages (barrières anti-bruit- murs ou capot anti poussière pour les bandes transporteuses, rideau végétal etc.). C'est ainsi le cas à Neuss où une halle a été construite pour limiter les pollutions atmosphériques et sonores d'une activité industrielle préexistante. L'antériorité n'est toutefois pas un gage de pérennité car le pouvoir électoral des résidents finit par peser plus lourd dans la balance que les considérations économiques ou l'antériorité. 
Lorsque les possibilités de redéploiement portuaire sont très contraintes, d'autres options doivent être envisagées pour des fonctions productives. Il y a d'abord la requalification des friches industrielles en privilégiant une activité liée au transport fluvial. C'est la solution privilégiée à Duisbourg après le démantèlement des sites sidérurgiques au profit d'activités logistiques (LogPort 1 et 2 ). Le succès a été tel que l'activité a essaimé sur l'ensemble des friches disponibles, parfois même sans accès direct aux voies navigables; ainsi le site de 15 hectares à Castrop-Rauxel à proximité du canal Rhein-Herne. Le choix de Francfort s'est porté sur le développement de type coopératif avec le port Gustavsburg qui dispose de réserves foncières. La densité des aménagements portuaires publics, en moyenne tous les 20 kilomètres le long du Rhin entre Bâle et Emmerich permet d'envisager des rapprochements et des complémentarités fonctionnelles hors des seules circonscriptions municipales.

La fusion des autorités portuaires voisines de Neuss et Düsseldorf (qui ont ensemble pris une participation de $45 \%$ dans le port de Krefeld) s'inscrit directement dans cette recherche d'économie d'échelle par la spécialisation des terminaux qui laisse envisager à terme l'urbanisation complète du site portuaire de Düsseldorf. Les recompositions en cours permettent d'imaginer à terme une gestion portuaire structurée autour d'une demi-douzaine d'organismes majeurs plus ou moins intégrés. Sur la base d'une étude de prévision (Planco 2013), la planification fédérale soutient au demeurant cette option autour des quelques grands pôles métropolitains.

Le mouvement de concentration des gestionnaires portuaires est aujourd'hui largement engagé (Beyer, 2013). Il prend différentes formes possibles de l'intégration au système d'accords de coopération plus lâche. La question des disponibilités foncières et les perspectives de reconversion urbaine entre largement en considération. Aussi, partant d'une situation de tension comparable et de la nécessité de trouver un nouvel arrangement entre Ville et Port, les acteurs locaux développent des solutions très variées. Des approches comparatives récentes confirment cette orientation en montrant comment cinq ports rhénans (Bâle, Strasbourg, Mannheim, Düsseldorf, Moerdijk) réévaluent la gouvernance de la relation avec les autorités urbaines et s'engagent à se positionner à de nouvelles échelles territoriales, qu'elles soient intra-ou interrégionales (Wite et al., 2016).

\section{Les rapports de force entre acteurs urbains et portuaires à la lumière des documents de planification en Allemagne}

Dans les ports majeurs, les municipalités contrôlent l'autorité portuaire et disposent indirectement d'un pouvoir de décision dans l'affectation du foncier portuaire (Tab. 2). Elles agissent sur deux tableaux : en tant que propriétaires des infrastructures via une société de gestion, mais aussi comme prescripteurs des documents locaux d'urbanisme. On peut donc considérer les villes comme juges et parties dans la question de réaménagement. Les acteurs portuaires redoutent ainsi que les choix se portent préférentiellement sur des opérations d'urbanisation rentables, d'autant que les Ports disposent d'emprises foncières centrales, d'un seul tenant et facilement mobilisables. Toutefois, le pouvoir du maire n'est pas absolu dans la mesure où les plans d'urbanisme doivent être compatibles avec les documents d'aménagement à l'échelle fédérale ${ }^{2}$ et surtout au niveau du Land. Si la prééminence municipale est la règle dans la gestion portuaire, certaines installations relèvent directement du Land (Rhénanie-Palatinat). 
Par ailleurs, si le pouvoir communal est établi dans les ports principaux, il n'est pas uniforme le long du Rhin ; il est plus étendu dans le Bade-Wurtemberg qu'en Hesse par exemple. Les enjeux économiques et environnementaux croissant de la voie d'eau dans la desserte des arrière-pays rhénans conduisent néanmoins à accroitre le pouvoir d'intervention de l'Etat fédéral et des Länder.

Tableau 2. La gouvernance des ports intérieurs allemands (d'après Stöckner, 2005)

\begin{tabular}{|l|l|l|l|}
\hline & Administration & Propriété & Exploitation \\
\hline Communes & 38,5 & 34,6 & 17,3 \\
\hline Länder & 7,7 & 13,5 & 3,8 \\
\hline Bund & 0 & 3,8 & 0 \\
\hline Privés & 34,6 & 32,7 & 50 \\
\hline Sociétés mixtes & 19,2 & 15,4 & 28,8 \\
\hline Total & 100 & 100 & 100 \\
\hline
\end{tabular}

Pour défendre les intérêts portuaires, les acteurs économiques et les associations fédérales pressent les Länder de suivre une politique plus active en vue de préserver le foncier portuaire. Dans ce domaine, le Land de Rhénanie-Westphalie (Ministerium NRW, 2008 et 2010) a mené une large réflexion, tant dans la définition d'une planification portuaire intégrée au plan régional de transport, que par des dispositifs juridiques visant à préserver les sites portuaires déjà classés. Entre l'initiative des Communes dans les questions d'urbanisme (Stadtentwicklungsplan) et le droit fédéral qui s'applique à la gestion de la voie d'eau, interviennent les documents de planification régionale (Raumordnung im Landesentwicklungsplan ou Regionalplan) (Erbguth \& Schubert, 2010) (Erbguth et Breuch-Moritz, 2012). Les réalisations urbaines en zone portuaire doivent se conformer aux règles générales d'urbanisme et de sécurité, notamment en ce qui concerne l'exposition aux émissions polluantes, motif qui a d'ailleurs été invoqué contre l'extension du projet d'urbanisation du port de Düsseldorf. En RhénanieWestphalie, le plan d'aménagement prescriptif (NRW Landesentwicklungsplan 2025) a permis de définir les implantations portuaires prioritaires (Hafenvorranggebiet). Le Land de NRW dispose ainsi d'importantes réserves foncières inscrites dans le plan régional de développement de 1978, mais à l'écart des aires métropolitaines ${ }^{3}$. Enfin, l'intervention des Länder au profit de l'activité portuaire peut aussi passer par des aides financières ciblées, susceptibles de soutenir les activités portuaires existantes ou leur développement.

Toujours sous l'égide du Land de NRW, une autre voie est possible, celle d'un aménagement intégré (integrierte Planung) entre les masters plans urbains et portuaires trop souvent pensés indépendamment l'un de l'autre. Cela suppose bien sûr une meilleure connaissance réciproque des acteurs et la définition négociée d'un projet local cohérent de moyen ou long terme. Dans ce cadre peuvent être envisagés des mécanismes de transfert d'affectations foncières, avec charge à la municipalité de 
compenser les zones urbanisées par un aménagement fluvial équivalent, afin de ne pas entamer le potentiel portuaire.

\section{Conclusion}

Le réaménagement des fronts portuaires qui s'est engagé dans les ports maritimes il y a plus de trente ans a progressivement touché les ports fluviaux, notamment ceux du Rhin. Plus que le retrait des activités lié à la conteneurisation et au gigantisme des navires, c'est ici la pression métropolitaine qui exerce un rôle décisif dans les mutations fonctionnelles. Les multiples projets qui s'égrènent de Nimègue à Bâle illustrent la convergence des ambitions et des formes que prend l'expression de la métropolisation sur les bords du Rhin. L'extraordinaire attrait du fleuve est un élément identitaire fort que partagent ces villes. Alors qu'elles avaient délaissé les berges aux activités industrielles et aux transports, elles réaffirment désormais leur ancrage rhénan. Nombreux sont les éléments qui s'inspirent des formes urbaines globalisées dont les waterfronts maritimes ont été le laboratoire. Les villes rhénanes s'alignent ici sur les attendus du marketing urbain international avec la promotion des effets de sites. Elles reproduisent l'idéal contemporain d'architecture tournée vers la performance économique et financière. Les formes urbaines s'inscrivent ici dans la continuité des fronts de mer. D'ailleurs les mêmes cabinets d'architectes sont sollicités dans les deux cas. Le contrôle public reste néanmoins plus étroit dans l'espace rhénan où les ports, les villes, les régions encadrent les processus à l'œuvre. On a donc à faire à un libéralisme tempéré.

L'ambition de disposer des nouveaux attributs métropolitains semblent entrer en conflit avec la position des acteurs portuaires qui voient leur position se fragiliser. Contraints dans leurs développements, les acteurs portuaires craignent désormais que les fonctions urbaines classiques ne finissent par étouffer l'activité portuaire ellemême. La menace est d'abord celle du transfert foncier effectif et irréversible au profit de la création des nouveaux quartiers centraux. La mutation est aussi plus insidieuse, car l'attribution de parcelles à des vocations résidentielles conduit aussi à des situations potentiellement conflictuelles entre les activités classiques implantées, souvent de longue date, et les nouveaux riverains particulièrement exigeants en termes de qualité environnementale. Le gestionnaire portuaire est ainsi amené à ménager des transitions entre espaces fonctionnels. Les redéfinitions successives des zones portuaires actives instillent une incertitude, qui liée aux nouvelles contraintes environnementales, font hésiter les investisseurs industriels et accentuent les risques de déprise. Toutefois, les opérations significatives touchent quasi-exclusivement des zones portuaires hyper centrales, largement désactivées du fait de leur enclavement. Rares sont en outre les fermetures sans compensation au sein de la circonscription municipale et de plus en plus à des échelles supérieures. Dans tous les cas l'évolution est accompagnée d'une négociation qui souligne la perméabilité entre acteurs urbains et portuaires et le souci de la recherche d'un juste équilibre. L'évolution des trafics et les ressources foncières effectives obligent donc à tempérer le discours alarmiste de certains acteurs portuaires.

Si certaines municipalités font le pari d'accompagner une conquête urbaine continue, la plupart des élus et des techniciens municipaux restent sensibles à l'atout que représente le port urbain en termes économique et industriel. On peut ici distinguer les 
centres majeurs où la suppression de l'activité est envisagée, les pôles urbains intermédiaires qui accompagnent le transfert vers des sites périphériques et enfin les pôles moins tendus qui sont en mesure d'accueillir un surcroît de trafic et des nouveaux terminaux. Par ailleurs, la mutation urbaine du port peut aussi être l'occasion de dégager des moyens financiers pour moderniser d'autres sites ou pour repenser l'organisation globale, de manière plus compacte et plus efficace, comme le rappellent les cas de Francfort ou de Bâle.

La question de la saturation des ports avec la croissance continue des trafics de conteneurs concerne surtout les hubs du Rhin inférieur (Duisbourg, Cologne, Düsseldorf, même si dans ce dernier cas des alternatives sont envisageables sur les sites de Godorf ou Reisholz. Le Land de Rhénanie-Westphalie dans les ports duquel transite la moitié des trafics fluviaux allemands est tout particulièrement sensible à cet enjeu. Par de nouvelles dispositions légales, il s'est engagé à assurer la préservation globale de son potentiel portuaire. Le développement des hubs qui articulent des espaces plus vastes ne peuvent que s'appuyer sur des plates-formes de redistribution bien connectées et largement dotées en capacité d'entreposage et de traitement logistique. Le gouvernement fédéral appuie fortement cette vision très hiérarchisée du système de transport fluvial (Planco, 2013). On est alors loin des espaces centraux ou péricentraux qui font actuellement l'objet d'une transformation urbaine.

Bibliographie

Beyer A., 2012, « Le port dans la négociation métropolitaine : l'exemple de Bâle », L'Espace géographique, $\mathrm{n}^{\circ} 3,2012, \mathrm{p} .252-265$.

Beyer A. (2013), Un panorama européen des stratégies de coopération interportuaires, Navigation ports \& intermodalités, 2013, p. 18-20.

Beyer A., Debrie J. (dir), (2014), Métropoles fluviales. Comment concilier développement urbain et logistique durable? Paris, L'Æil d'Or, 2014, 317 p.

Bird J., 1971, Seaports and Seaport Terminals, London, Hutchinson, 240 p.

BÖB, 2006, Bundesverband Öffentlicher Binnenhäfen, Binnenhäfen zwischen Wachstumsmotor und Bedeutungsverlust. Hafen und Stadtentwicklung in einem stabilen Gleichgewicht, Argumentationspapier, Berlin, 26 p.

BÖB, 2007, Bundesverband Öffentlicher Binnenhäfen, Hafen und Stadtentwicklung in einem stabilen Gleichgewicht, Sonderthemendienst zum Parlamentarischen Abend am 1. Februar 2007 im Rathaus zu Berlin, Berlin, 14 p.

Breen A., Rigby A., 1996, The New Waterfront: A Worldwide Urban Success Story, London, Thames and Hudson, $224 \mathrm{p}$

Brownhill S., 2013, «Just Add Water. Waterfront Regeneration as a global Phenomenon » in Leary M. E., Carthy J. (dir.), (2013), The Routledge Companion to Urban Regeneration, Londres, Routledge, p. 45-55.

Chaline C., Rodrigues Malta R., 1994, Ces ports qui créèrent des villes, Paris, L'Harmattan, 299 p.

Charles Jencks, 1984, What is Postmodernism? London, Academy Editions, 152 p.

Charlier J., 2007, « Les enjeux des redéveloppements ville-port », Programme cadre régional HANSE PASSAGE, «Faire la ville avec le port. Quelles stratégies pour le redéveloppement des espaces de liaison ville/port? Guide des bonnes pratiques », Le Havre, 136 p. 
Dablanc L., Frémont A. (dir), 2016, La métropole logistique. Le transport de marchandises et le territoire des grandes villes, Paris, Armand Colin, $320 \mathrm{p}$.

Debrie J., Raimbault N., 2016, « The port-city relationships in two European inland ports: a geographical perspective on urban governance », Cities - International Journal of Urban Policy and Planning, n50, p.180-187.

Dyson K., Yocom K., 2015, « Ecological design for urban waterfronts », Urban ecosystems, Volume 18, Issue 1, p.189-208.

Erbguth W., Breuch-Moritz M., 2012, Anlage und Erweiterung von Häfen: Rechtsfrage. Rostocker Infrastrukturrechttag 2011, Baden-Baden, Nomos, $102 \mathrm{p}$.

Erbguth W., Schubert M., 2011, Rechtsfragen der Errichtung und Erweiterung von Binnenhäfen unter Berücksichtigung städtebaulicher Nutzungsinteressen an Hafenflächen, Baden-Baden, Nomos, 185 p.

Fläming H., Hesse M., 2010, « Binnenhäfen: wachsen oder weichen? », Raumplanung, 149, p. 97-101.

Frémont A.,2012, «Quel rôle pour le fleuve dans le Grand Paris des marchandises ? », L'Espace géographique, 2012/3 (Tome 41), p. 236-251.

Gintrac C. et Giroud M., 2014, Villes contestées : pour une géographie critique de l'urbain, Paris, Les Prairies ordinaires, $399 \mathrm{p}$.

Hall P, Clark A., 2010, «Maritime ports and the politics of reconnection», in Desfor G. et al. (Coord.), Transforming urban waterfronts: fixity and flow, New-York, Routledge, p. 17-34.

Hendricks B., 2017, « Immer weniger Sozialwohnungen », Die Zeit, édition du 22/2/2017.

Holländer R., Kochmann L., Völkner T. Strauss, C., 2011, Erfolgreiche Transformation industrialisierter Flussgebiete. Erfahrungen aus der europäischen Praxis. Selbstverlag, Leipzig, 169 p. Höhmann I., 2010, « Rheinauhafen Köln: Vorzeigequartier wartet auf Anschluss », Frankfurter Allgemeine Zeitung, 20.11.2010.

Hoyles B.S., 1989, « The port-city interface: trends, problems and examples », Geoforum, 20, p. 429-435.

Jencks C., 1977, The Language of Postmodern Architecture, New York, Rizzoli, 104 p.

Malone P. (Ed.), 1996, City, Capital and Water, London, Routledge, 274 p.

Marshall R. (dir), 2001, Waterfronts in post-industrial cities, London \& New York, Spon Press, 194 p.

Merckx F., NotteboomT., W Winkelmans W., 2003, Spatial models of waterfront redevelopment: the tension between city and port revisited, Proceedings of IAME, $19 \mathrm{p}$.

Michon P., 2008, «L'opération de régénération des Docklands : entre patrimonialisation et invention d'un nouveau paysage urbain », Revue Géographique de l'Est [En ligne], vol. 48 / 1-2 | 2008, mis en ligne le 09 octobre 2011, consulté le 09 mai 2016. URL: http://rge.revues.org/1104.

Ministerium für Bauen und Verkehr des Landes Nordrhein-Westfalen, 2008 Wasserstraßenverkehr, Binnenhäfen und Logistik in Nordrhein-Westfalen Fortschreibung des Wasserstraßenverkehrs- und Hafenkonzeptes Nordrhein-Westfalen, Düsseldorf, 66 p.

Ministerium für Wirtschaft, Energie, Bauen, Wohnen und Verkehr des Landes NordrheinWestfalen, 2010, Binnehäfen im Spannungsfeld konkurriender Nutzungsinteressen, Düsseldorf, 65 p.

Paffoni E., 2013, Renouveau du transport fluvial et dynamiques métropolitaines : le cas des ports fluviaux franciliens (1980-2010), thèse de géographie, Université Paris Est, Marne-la-Vallée, 322 p. 
Planco, 2013, Gutachten zur Erhöhung der Wettbewerbsfähigkeit der Binnenhäfen, Endbericht, Essen, BMVBS, $170 \mathrm{p}$.

Ponzini D., Nastasi M., 2011, Starchitecture: Scenes, Actors and Spectacles in Contemporary Cities. Turin, Allemandi, 2011, 144 p.

Raimbault N., 2014, Gouverner le développement logistique de la métropole : périurbanisation, planifi cation et competition métropolitaine, le cas du bassin parisien et éclairages étrangers, Thèse, Marne-laVallée, Université Paris-Est, 524 p.

Sassen S., 1996, La ville globale. New York, Paris, Descartes et Cie, 533 p.

Schubert D., 2011, Transformationsprozesse in Seehafenstädten - Revitalisierung von (brachgefallenen) Hafen- und Uferzonen, Bibliography (Seaport Cities and Waterfront Revitalisation), Updated Version: Juli 2011, en ligne.

Sepe M., 2013, « Urban history and cultural resources in urban regeneration: a case of creative waterfront renewal », Planning Perspectives, p. 595-613.

Smith N., 2002, « New Globalism, New Urbanism: Gentrification as Global Urban Strategy », Antipode, Volume 34, Issue 3, p. 427-450.

Stadt Köln, 2016, Beschlussvorlage zur Behandlung in öffentlicher Sitzung Betreff Kooperatives Verfahren Deutzer Hafen, 15/12/2016, 4 p.

Stein Sandra, 2012, Logistikpark Binnenhafen. Geographie in der Praxis Bd. 5, Berlin, Uni-edition, $233 \mathrm{p}$.

Stöckner U., 2005, Strategien zur Revitalisierung von Hafenarealen im Binnenland - aufgezeigt am Beispiel der Rhein- und Neckarhäfen, Dissertation, Karlsruhe, 193 p.

Ville de Strasbourg, 2010, « Port du Rhin : un quartier totalement renouvelé », Lettre des deux rives, mars 2010 .

Wang C., 2002, Waterfront Regeneration, Cardiff, Cardiff University, 2002, 234 p.

Wiegmansa B. W., Louwa E., 2011, « Changing port-city relations at Amsterdam: A new phase at the interface? ». Journal of Transport Geography. Volume 19, Issue 4, July 2011, p. 575-58.

Witte, P.A., Wiegmans, B.W., van Oort, F.G., Spit, T.J., 2016, « Weakest link or strongest node? Comparing governance strategies for inland ports in transnational European corridors, Research in Transport Business and Management » $\mathrm{n}^{\circ} 19$, p. 97-105.

\section{NOTES}

1. Le terme de douane couramment utilisé renvoie au statut d'entrepôts et de port francs dont jouissaient nombres d'installations. Le Rhin, voie d'eau internationale, permettait l'importation de denrées, notamment coloniales mais aussi de produits de luxe, en franchise de douane.

2. En fait, l'Etat fédéral centre son intervention sur la gestion de la construction et de l'entretien de la voie d'eau. Il n'intervient pas ou exceptionnellement dans la question des aménagements portuaires eux-mêmes, sauf en cas de création de nouvelles installations portuaires qui viennent alors impacter le dispositif hydrographique existant. 
3. Une dizaine de sites bord à quai, de taille importante (plus de 200 ha) avait été réservée à l'implantation de grandes unités industrielles, d'une taille minimale unitaire de 200 ha (surfaces ramenées à 80 ha en 1995).

\section{RÉSUMÉS}

L'article rend compte d'une évolution commune aux métropoles rhénanes, celle de l'urbanisation progressive des emprises portuaires au profit de réalisations architecturales ambitieuses. Cette dynamique met de fait en relief les concurrences spatiales entre deux facettes de la métropolisation dans laquelle s'inscrivent ces espaces: d'une part, la mutation des espaces portuaires centraux en zones résidentielles de prestige, aptes à retenir ou accueillir les fonctions de commandement et les cadres que souhaitent attirer les édiles ; d'autre part, la nécessité de préserver les équipements portuaires pour répondre à une pression logistique croissante, vecteur de l'insertion de l'économie rhénane dans la globalisation. Partant de la figure du waterfront maritime, l'analyse montre les ressorts de ces mutations et de quelle manière les adaptations locales qui en résultent permettent de différencier des configurations urbaines. Les différentes situations analysées soulignent que les mutations ne remettent pas fondamentalement en cause les fonctions de transport, crainte portant régulièrement soulevée par les autorités portuaires.

The paper gives an insight into the common urban development of the major cities along the Rhine. It pinpoints the central role of urban conversion of former port areas into ambitious development zones. The local dynamics is based on the spatial competition between two contradictory metropolitan tendencies: on the one hand, the search for prestigious residential dwelling on the river banks in a central location to attract CBD-functions; on the other hand, the need to preserve port equipment under increasing logistical pressure. A first discussion shows to what extend the socio-spatial model of the maritime waterfronts can be applied to the urban situation of Rhenish cities as the dynamic largely observed in sea ports for the 30 last years is now concerning river ports. The numerous examples of new and attractive projects from Nijmegen to Basel illustrate a large consensus on urban port evolution as a central issue for urban redevelopment and expansion of the city core functions that lead to the construction of a morphological and dynamic typology of the Rhine ports. In most of the cases, the prestigious buildings are the ultimate showcase of a new way of living and promoting contemporary urbanity. However, the quantitative need and offer of land for port activities may temper the excessively alarmist position of the river port lobbies.

Der Artikel versucht einen genauen Überblick über eine gemeinsame räumliche Entwicklung widerzugeben, die die gegenwärtigen Rheinmetropolen gekennzeichnet: die rasche Urbanisierung der city-nahen Hafengebieten durch anspruchsvolle stadtbauliche Programme. Diese Dynamik deutet auf die zähen Wettbewerbsverhältnisse zwischen zwei konkurrierende Aspekte der Metropolisierung: zum einen die Anforderung auf hochqualitativen Wohngebieten, um wirtschaftliche Führungsfunktionen und privates Kapital anzuziehen, und zum anderen, die Notwendigkeit, Hafeninfrastruktur aufrecht zu erhalten, um auf den zunehmenden logistischen Druck reagieren zu können. Nach der Erörterung des „maritim waterfront“-Begriffes, dass man an den Rheinmetropolen gut anwenden kann, deutet die Analyse auf die Ursachen und Formen raumplanerischer Umwandlungen. Sie stellt fest, dass ihre negativen Auswirkungen auf die 
Hafenaktivität oft überschätzt sind. Anhand verschiedener Fallbeispiele, wird es gezeigt, dass die Vielfalt der lokalen Umsetzungen große Bedeutung zukommt.

\section{INDEX}

Schlüsselwörter : Rhein, Fluss, Binnenhafen, städtische Umstrukturierung, städtebauliches Modell, Waterfront

Keywords : Rhine, river, port, urban regeneration, waterfront, urban model

Mots-clés : Rhin, fleuve, ports, régénération urbaine, waterfront, modèle urbain

\section{AUTEUR}

\section{ANTOINE BEYER}

laboratoire MRTE, Université Cergy-Pontoise

Site des Chênes - 33 bd du Port 95011 Cergy-Pontoise cedex.

Antoine.beyer@u-cergy.fr 